\title{
Les formes élémentaires, ou la naissance d'une socio-anthropologie symbolique
}

The Elementary Forms of Religious Life, or the birth of a symbolic socio-anthropology

\section{Las formas elementales o el nacimiento de una socio-antropología simbólica}

\section{Stéphane Vibert}

Numéro 56, printemps 2014

Émile Durkheim : généalogie, critique et épreuve

URI : https://id.erudit.org/iderudit/1031377ar

DOI : https://doi.org/10.7202/1031377ar

\section{Aller au sommaire du numéro}

\section{Éditeur(s)}

Athéna éditions

ISSN

0831-1048 (imprimé)

1923-5771 (numérique)

Découvrir la revue

Citer cet article

Vibert, S. (2014). Les formes élémentaires, ou la naissance d'une socio-anthropologie symbolique. Cahiers de recherche sociologique, (56), 131-141. https://doi.org/10.7202/1031377ar
Résumé de l'article

Si les thèses développées dans les Formes élémentaires de la vie religieuse ont subi bon nombre de critiques depuis sa parution, tant au niveau de la validité des faits relevés que de l'interprétation du totémisme, et si la théorie

" sociologique » de l'essence de la religion continue évidemment de faire débat, il n'en reste pas moins que la question la plus centrale de l'ouvrage - et la plus actuelle puisqu'elle oriente la compréhension même du rôle et de la nature des sciences sociales - s'avère d'ordre ontologique : l'intelligence de la société comme réalité tant idéelle que matérielle, individuelle que collective, bref comme réalité symbolique. À partir notamment des lectures stimulantes de Karsenti et Tarot, le symbolisme sera présenté comme logique spécifique des représentations collectives, comme processus par lequel un groupe humain, tout en s'ordonnant autour d'un ensemble structuré d'idées-valeurs, ne se conçoit comme totalité concrète que par la circulation de formes sensibles maintenant les acteurs en interdépendance relationnelle. 


\section{Les formes élémentaires, ou la naissance d'une socio-anthropologie symbolique}

StéPHANE ViberT

$\mathbf{N}$ ombre de lecteurs l'ont signalé, Les formes élémentaires de la vie religieuse s'avèrent un ouvrage d'une complexité interne redoutable. Sa puissance et sa difficulté qui, à bien des égards, sous-tendent son caractère de livre "classique ", font qu'il se voit constamment repris, discuté, critiqué, amendé, annexé - comme encore récemment par Ann Rawls sous la forme d'un «socio-empirisme ${ }^{1}$ »-, alors même que certaines thèses de fond, à commencer par la lecture du totémisme qui en constitue pourtant apparemment le centre, semblent irrémédiablement datées ${ }^{2}$. À la suite de sa lecture des Formes élémentaires, Camille Tarot a trouvé dans l'ouvrage au moins sept grands thèmes, tous plus ou moins susceptibles d'être interrogés, débattus ou développés de façon autonome ${ }^{3}$ : la définition de la religion par l'opposition sacré/profane; la critique des autres théories existantes de la religion; la présentation du totémisme australien comme forme religieuse élémentaire; la proposition d'une théorie sociétiste de la religion, selon une explication à la fois positive et générale de son origine; l'introduction d'une sociologie de la connaissance fondée d'une part sur l'origine sociale des

1. Ann W. Rawls, «La théorie de la connaissance de Durkheim, un aspect négligé de son œuvre», dans Michel de Fornel et Cyril Lemieux, Naturalisme versus constructivisme?, Paris, Éd. de l'E.H.E.S.S., 2007, p. 58.

2. Frederico Rosa, L'Âge d'or du totémisme. Histoire d'un débat anthropologique (1887-1929), Paris, C.N.R.S. ÉditionsÉd. de la Maison des Sciences de l'Homme, 2003.

3. Camille Tarot, De Durkheim à Mauss, l'invention du symbolique, Paris, La Découverte et Syros, 1999, p. 211. 
catégories religieuses et, d'autre part, sur l'origine religieuse des catégories philosophiques et scientifiques; une théorie des rites; et enfin, septième et dernier grand thème, non le moindre dans le contexte contemporain, une appréciation argumentée sur le devenir de la religion dans l'histoire de la civilisation, particulièrement quant à l'éventuelle sécularisation et la résurgence des phénomènes dits religieux dans la modernité.

Ce n'est pourtant pas l'un de ces sept thèmes qui sera l'objet de ce texte, mais une question transversale, qui apparait pour ainsi dire implicitement dans le développement de l'ouvrage. Je veux parler de cet immense espace d'intelligibilité que Tarot a nommé l'invention du symbolique, invention que l'on peut associer à la naissance d'une véritable socio-anthropologie symbolique, pour marquer combien il s'agit non seulement d'une découverte conceptuelle, mais surtout d'une révolution intellectuelle, tant ontologique, épistémologique que méthodologique. Nous sommes donc en présence d'un moment qui influence irrémédiablement l'évolution des sciences sociales jusqu'à nos jours, et nous permet de considérer l'humain d'un regard renouvelé. Non pas tant que cette perspective fasse hier comme aujourd'hui l'unanimité, loin s'en faut. Mais l'on peut suggérer qu'il s'agit de l'une des voies les plus fécondes à la fois pour appréhender la nature culturelle de l'homme - ce niveau d'être ontologique que Castoriadis appelle le «socialhistorique ${ }^{4}$ », en tant que réalité synthétique a priori et sui generis -, et à la fois afin de décrire et de comprendre les modalités concrètes de cette existence sociale - selon ce que le philosophe Vincent Descombes, interprétant l'héritage de Montesquieu, Durkheim, Mauss ou Louis Dumont, a désigné comme un «holisme structural ${ }^{5}$ », voué à incarner la véritable "science des institutions» pensée par Durkheim et Mauss.

Nous présenterons tout d'abord succinctement les circonstances théoriques qui vont conduire Durkheim à circonscrire la notion de «symbole » pour l'ériger en point central de son questionnement, puis - dans un deuxième temps - la façon dont cette réflexion débouche sur un déplacement majeur pour la discipline naissante qu'est la sociologie, en favorisant la naissance de ce que tant Camille Tarot que Bruno Karsenti ont interprété, à la lecture des Formes élémentaires, comme "une véritable théorie du symbolisme ${ }^{6}$ ». Nous nous appuierons d'ailleurs largement de façon explicite sur leurs écrits tout au long de cette argumentation, afin d'aboutir à un dernier temps conclusif, au cours duquel il s'agira de préciser en quoi cette avancée décisive pour la compréhension socio-anthropologique ouvre, tant pour Mauss que pour

4. Cornelius Castoriadis, L'institution imaginaire de la société, Paris, Seuil, 1975.

5. Vincent Descombes, Les institutions du sens, Paris, Éd. de Minuit, 1996.

6. Bruno Karsenti, L'homme total. Sociologie, anthropologie et philosophie chez Marcel Mauss, Paris, PUF, 1997, p. 210. 
d'autres, un «champ des possibles» immense, toujours accessible et fécond, qui reconfigure la conception du symbolisme situé au cœur de la vie sociale, tout en renouvelant par-là décisivement l'intelligence de celle-ci.

\section{La question du symbole dans la vie sociale}

Tant dans la philosophie que dans les sciences sociales du XIX' siècle, l'interrogation sur le symbole s'ancrait avant tout dans une réflexion à propos du symbolisme religieux, sous l'impulsion notamment des travaux de Creuzer, repris par Hegel, le premier à avoir étudié rigoureusement les représentations mythologiques non pas d'après leur valeur esthétique ou imaginaire, mais en cherchant à mettre en lumière la rationalité intrinsèque de leur signification. Chez un Renan également, on trouve déjà la thèse de l'indissociabilité dans le phénomène religieux du symbole et du dogme, de la parole et de la pensée, de l'expression et de l'idée, tout en admettant bien entendu le fait que le symbole soit, par nature, «insuffisant et condamné à rester bien au-dessous de l'idée qu'il représente ${ }^{7} »$. Appliqué au christianisme dans le monde occidental, le symbole constitue ainsi un signe au moyen duquel les membres appartenant à l'Église en tant que communauté savent se reconnaître, attestant de l'existence d'une entité collective unifiée par le dogme. Il apparait évident que cette "conception juridico-religieuse ${ }^{8}$ » va très fortement influencer la manière dont Durkheim fait référence au terme de symbole. Dès sa thèse sur La division du travail social, en effet, dans le but de lire le mode de solidarité propre à une société à travers son système juridique, il considérait déjà le droit comme le «symbole visible de la socialité ${ }^{9}$ ", l'institution par laquelle celle-ci s'incarne, se divulgue et se concrétise. Ainsi, écrit-il,

... la solidarité sociale est un phénomène tout moral qui, par lui-même, ne se prête pas à l'observation exacte ni surtout à la mesure. Pour procéder à cette quantification tant qu'à cette comparaison, il faut donc substituer au fait interne qui nous échappe un fait extérieur qui le symbolise et étudier le premier à travers le second. Ce symbole visible, c'est le droit ${ }^{10}$.

Or, lorsque cette démarche se voit appliquée aux sociétés traditionnelles à solidarité mécanique, distinguées par un droit pénal de type répressif fondé sur l'expiation, Durkheim constate que «sous quelque symbole qu'elle se fasse sentir aux consciences", c'est "une puissance, en quelque sorte, transcendante ${ }^{11}$ qui se trouve à la base de l'édifice normatif. "C'est que la reli-

\footnotetext{
7. Renan, cité dans B. Karsenti, L'homme total, op. cit., p. 208.

8. Ibid., p. 208.

9. Ibid., p. 209.

10. Émile Durkheim, De la division du travail social, Paris, PUF, coll. «Quadrige», 1991, p. 28.

11. Ibid., p. 112.
} 
gion y pénètre toute la vie juridique, comme d'ailleurs toute la vie sociale ${ }^{12}$ », peut-il alors en conclure.

Ainsi, dès son ouvrage sur le suicide, Durkheim aura une vision claire de la religion, dont les définitions ultérieures resteront, par bien des aspects, étonnamment proches: "La religion, c'est en définitive le système de symboles par lesquels la société prend conscience d'elle-même; c'est la manière de penser propre à l'être collectif ${ }^{13}$.» Les formes élémentaires vont consacrer cette reconnaissance d'une primauté accordée à la religion, décrite, dès 1899 , comme le germe d'où sont issues toutes les autres sphères du social, contenant «en elle, dès le principe, mais à l'état confus, tous les éléments qui, en se dissociant, en se déterminant, en se combinant de mille manières avec eux-mêmes, ont donné naissance aux diverses manifestations de la vie collective $^{14} »$. Néanmoins, il faut remarquer dans cette analyse généalogique un déplacement par rapport à l'analyse juridico-religieuse propre à l'histoire chrétienne: en faisant du totémisme, religion sans écriture, une religion du symbole, Durkheim va séparer «le symbole de la formule énonciative pour le rattacher au signe dans sa matérialité même $\mathrm{e}^{15}$ ». De ce fait, le symbole ne tient plus son efficacité du contenu de vérité révélée qu'il transporte et exprime, mais du simple fait qu'il signifie pour chacun et pour tous, «intégré à un processus de communication constitutif de toute entité collective ${ }^{16} »$. Incontestablement, ce basculement fonctionnaliste va contribuer à défaire l'idée selon laquelle la notion de divinité incarne la dimension centrale et constitutive d'une essence de la religion. Loin désormais d'en représenter le soubassement conceptuel majeur, la divinité se retrouve déplacée vers la périphérie du religieux comme fait social, puisque reconsidérée en termes de symbole, plus ou moins contingent, de la vie collective elle-même, et en tout état de cause, comme telle nullement indispensable au final. Réévaluée à l'aune de son rôle social, d'écrire Durkheim, «l'idée de Dieu qui tout à l'heure semblait être le tout de la religion ne devient plus qu'un accident accessoire », qui, en fin de compte, a surtout «servi à symboliser toutes sortes de traditions, d'usages, de besoins collectifs ${ }^{17}$ ", ce qui constitue la base cachée, mais réelle de la religion, puisqu'elle trouve son origine dans la société.

Ce passage fondamental du contenu à la fonction constitue, on le sait, la ligne de démarcation construite par Durkheim afin de différencier la sociolo-

\footnotetext{
12. Id.

13. Émile Durkheim, Le suicide, Paris, PUF, 1979, p. 352.

14. É. Durkheim (dans la Préface du second volume de L'Année sociologique, daté de 1899), cité dans B. Karsenti, L'homme total, op, cit.., p. 210.

15. Ibid., p. 208 .

16. Id.

17. É. Durkheim, cité in C. Tarot, De Durkheim à Mauss, op, cit.., p. 218.
} 
gie de l'histoire des religions et de la théologie. Le sociologue doit se borner à étudier le «rôle social» de la religion, d'autant plus que toutes les religions, y compris dans leurs aspects les plus personnels et les plus mystiques, restent «sous la dépendance du groupe sans le savoir ${ }^{18}$ ». Si l'expérience religieuse, comme d'ailleurs toutes les croyances, se trouve nécessairement reliée à des conditions historiques, sociales et culturelles, alors la religion joue essentiellement un rôle moral, en un double sens : d'une part, "elle s'inscrit dans la réalité des mœurs» et, d'autre part, elle fournit «des idéaux, des normes pratiques pour orienter les comportements ${ }^{19}{ }^{\prime}$. D'où sa définition, bien connue, comme un «ensemble plus ou moins complexe de mythes, dogmes, rites, cérémonies et opinions» qui ont la particularité d'être obligatoires, à partir d'une distinction universelle du sacré et du profane, mais toujours incarnée originalement dans un groupe défini. Tarot a rappelé qu'il y avait eu, dans le contexte de l'Affaire Dreyfus, un court moment dans lequel Durkheim avait semblé défendre la possibilité d'une «religion purement morale ${ }^{20}$ ", complètement spiritualisée et dépourvue de médiations sociales. Or, ce n'est finalement pas du tout la forme que va prendre la théorie durkheimienne, qui entend au contraire insister sur la nécessité structurale du processus de symbolisation au cœur du phénomène religieux. Ce processus de symbolisation, présenté de façon complexe dans les Formes élémentaires, prend une double orientation, à la fois causaliste et fonctionnelle, qui finit par se focaliser sur la mise en évidence du totem comme emblème religieux de la société pour elle-même.

La dimension explicative repose sur ce que Tarot a nommé la «théorie dynamogénique de la religion ${ }^{21}$ », hypothèse selon laquelle les représentations religieuses - non pas simple reflet de la réalité extérieure mais poussées opérantes, principes actifs d'idéalisation - reposent en dernière instance sur un système de forces se projetant sur le monde. Un système de forces qui relève avant tout de l'inconscient, et qui se traduit par des habitudes collectives composées de sentiments, d'affects, d'émotions ou de besoins, lesquels se seraient éveillés d'eux-mêmes, spontanément, en continuité avec les sociétés forgées au sein du monde animal évolué. Mais ce véritable schéma causal de l'origine ne saurait se passer d'une explication fonctionnelle, selon laquelle ce groupe naturellement donné, encore inconscient de lui-même, doit, pour devenir véritablement ce que nous appelons une "société", canaliser son énergie collective, notamment lorsqu'il entre en effervescence, en la diri- 
geant sur des signes matériels appelés à représenter son unité et à l'inscrire dans la durée. C'est pour cette raison qu'ils seront alors destinés à incarner la dimension sacrée de la société.

On sait qu'étudiant les Aborigènes australiens, considérés en son temps comme société la plus primitive de l'espèce humaine, Durkheim verra dans l'emblème totémique "la preuve que le symbolisme est au cœur du phénomène religieux et par conséquent du social ${ }^{22}{ }$. Si le symbole est pensé sous le mode de l'emblème, c'est bien parce que les sentiments collectifs ont pour nécessité de s'incarner dans des "emblèmes matériels", qui en deviennent les représentations figurées, tout comme peuvent l'être également parfois, de préciser Durkheim, des personnes particulières ou des formules rituelles. La religion met en jeu certaines fixations matérielles des forces sociales qui, sinon, resteraient à l'état diffus et inefficace. Parce qu'elles sont collectives, ces forces affectives ont à être symboliquement instituées, et cela passe par une forme extérieure visible de tous: «La conscience que la société prend d'elle-même dans les pratiques et croyances religieuses trouve donc dans le symbole son medium essentiel ${ }^{23}$.»

\section{La théorie durkheimienne du symbolisme et son évolution}

Pour Tarot comme pour Karsenti, dont cette présentation suit la ligne de pensée commune, il est clair que la conception durkheimienne du symbolisme subit une inflexion importante, pour ne pas dire davantage, à mesure de l'importance accrue accordée par le sociologue au fait religieux dans la consistance du lien social. Dans de nombreux passages en effet, à l'aune d'une approche sommairement fonctionnaliste du religieux, est attestée une compréhension du symbole de nature foncièrement représentative, c'est-àdire qu'il demeure envisagé comme «un processus figuratif de substitution d'une réalité à une autre ${ }^{24} »$. Certaines formulations de Durkheim laissent à penser que, durant un certain temps, selon une théorie dite sociétiste de la religion, il défend l'idée d'un rapport asymétrique entre le réel, associé à la morphologie sociale quant à sa constitution sensible, et le symbole, qui n'en serait qu'une expression dérivée, à contourner et interpréter afin de remonter à la véritable cause effective du phénomène religieux. "À ce niveau, d'écrire Tarot, le social n'est pas et ne peut pas être symbolique en lui-même, car il est la réalité et le symbole n'est qu'une manière de le dire, une figure, une traduction plus ou moins trompeuse, un travestissement ${ }^{25}$.»

22. Ibid., p. 221.

23. B. Karsenti, L'homme total, op. cit., p. 211.

24. Ibid., p. 210.

25. Camille Tarot, Le symbolique et le sacré. Théories de la religion, Paris, La Découverte, 2008, p. 264. 
Dans les diverses conceptions du symbole que l'on retrouve couramment dans les écrits durkheimiens, trois acceptions ${ }^{26}$ font essentiellement signe vers cette réalité seconde et illusoire, que l'on n'hésiterait pas à appréhender, pour bien en montrer la fonction potentiellement aliénante, comme «idéologique». Ce sont des passages qui font emploi de la notion de symbole comme synonyme d'allégorie ou métaphore, donc de revêtement superficiel, pour le premier usage; de symptôme, donc de traduction d'un dysfonctionnement pathologique par rapport au mouvement normal des choses, pour le deuxième usage; et enfin, un troisième usage fait référence au symbole avant tout comme cristallisation, ou condensation de pratiques et d'idées. Dans tous les cas, cette définition initiale du symbole reste dans l'orbite de la catégorie des «représentations», fussent-elles collectives, comprises comme «représentation ", présentation à nouveau, donc seconde et auxiliaire, exprimant une réalité qu'elle ne fait pas mais qu'elle reflète. Cette réalité du social qui résiderait avant tout dans un tissu d'interdépendance conçu comme le véhicule des affects, des sentiments et des passions qui en sont la force motrice et la cause originaire. Nous en restons alors à ce que l'on pourrait nommer une définition restreinte du symbole, en tant que «forme de substitution à la réalité ${ }^{27}$.

Toute la puissance et la fécondité des Formes élémentaires tient justement au fait que la théorisation du symbole ne s'arrête pas à ce point, pourtant déjà fondamental, et que la thèse ouvre - au-delà de la simple reconnaissance de l'importance du symbolisme dans la vie sociale - sur une véritable conception symbolique de l'être social, laquelle évidemment emporte des conséquences immenses sur le type de socio-anthropologie qu'il peut nous être donné de pratiquer, afin d'avoir accès à la vision la plus juste possible du niveau ontologique cohérent et autonome qu'est la société. On peut à cet égard montrer à la fois comment se réalise théoriquement cette opération d'extension de la logique symbolique qui, si elle n'est sans doute pas complète chez Durkheim, permet au moins d'indiquer les lignes directrices de son effectuation, mais également ce qu'elle permet au niveau épistémologique, entre autres la possibilité d'échapper à l'opposition binaire entre empirisme et idéalisme, opposition qui continue d'ailleurs de diviser les lectures faites de l'œuvre durkheimienne. Reprenons sommairement ces deux aspects.

Du côté théorique, il ne serait pas faux d'affirmer que dans quelques passages des Formes élémentaires se trouve l'intuition géniale, qui sera particulièrement exploitée et approfondie par Mauss, d'une extension compréhen-

26. Ibid., p. 283.

27. B. Karsenti, L'homme total, op. cit., p. 217. 
sive du symbolisme, en le dégageant de son ancrage kantien dans la nature des représentations. Car alors, à certains endroits, le symbolisme acquiert sa consistance propre en abandonnant son statut de vecteur second d'une réalité sociale à fondement morphologique, pour devenir la condition et l'expression même de l'existence ontologique du social, désormais compris comme idéation pratique ${ }^{28}$. Durkheim en vient ainsi à envisager l'hypothèse, notamment dans le cas du clan (pourtant pensé initialement à l'articulation de la nature et de la culture), que, sans le symbolisme, le social n'existerait sans doute pas. Sans le nom et le signe qui le matérialisent, écrit Durkheim, «le clan n'est même plus représentable ${ }^{29} »$. C'est pourquoi en se gardant de voir dans les symboles «de simples artifices» ou «des sortes d'étiquettes qui viendraient se surajouter à des représentations toutes faites $»^{30}$, Durkheim se déplace subrepticement mais incontestablement vers une conception du symbolisme comme dimension centrale et constitutive de la vie sociale, qui, écrit-il, «sous tous ses aspects et à tous les moments de son histoire, n'est possible que grâce à un vaste symbolisme ${ }^{31}$ ».

Durkheim en arriverait par là «à cette thèse essentielle de la nécessité fonctionnelle de la symbolisation ${ }^{32}$ », celle-ci rendant possible non seulement la prise de conscience par une société de son existence et de son unité, à partir des unités amorphes et massives de la horde primitive, mais incarnant surtout son identité et ses frontières, à travers l'énonciation d'une origine et d'une mémoire collective. Cette "théorie de la symbolisation» émergente dans les Formes élémentaires est aussi attestable par le fait que, au cours de la vie sociale, le totem devient finalement plus sacré que l'espèce réelle qu'il représente, et que le symbolisme se révèle d'autant plus nécessaire et incontournable que tout ou presque parait à Durkheim susceptible d'être sacralisé, de façon quasi arbitraire. Il faudrait donc en conclure que le symbolisme humain ne s'ajoute pas à la société, mais qu'au contraire, puisque c'est lui qui «rend compte du caractère d'où Durkheim avait déduit la conscience collective, l'extériorités ${ }^{3}$ ", il doit être saisi comme inhérent au fait social, à titre de condition incoercible.

28. «Ce qui fait la réalité, en tant que réalité de croyance et de pensée [...], ce n'est pas une idée intangible, ni une puissance idéelle capable de produire la réalité intégralement, au point que le postulat d'un donné indépendant des représentations deviendrait inconsistant. C'est un processus d'idéalisation des choses, par quoi elles se transforment. Un tel processus est créatif en un certain sens, et il l'est dans la mesure où il est collectif»: Bruno Karsenti, «Une alternative au-delà du pragmatisme. La pratique en suspens», dans Michel de Fornel et Cyril Lemieux, Naturalisme versus constructivisme?, op. cit., p. 138.

29. É. Durkheim, Les formes élémentaires de la vie religieuse, Paris, PUF, coll. «Quadrige», 2005, p. 334.

30. Ibid., p. 331.

31. Id.

32. C. Tarot, De Durkheim à Mauss, op. cit., p. 223.

33. C. Tarot, Le symbolique et le sacré, op. cit., p. 287. 
Par la double face du symbole, tant unité matérielle qu'événement psychique, «le social s'affirme à la fois et indissolublement comme chose et comme représentation $^{34}$ », ne se réduisant donc ni à l'un ni à l'autre. Dans ce cadre, le symbole n'est donc plus seulement un medium, autrement dit un procédé commode et instrumental qui rendrait plus clair le sentiment que la société a d'elle-même, mais un élément définitivement constitutif dans la conscience réflexive de la société, la modifiant justement en retour dans sa réalité concrète et l'existence vécue de ses membres.

Cette intuition théorique se double d'une ouverture épistémologique considérable. Longtemps, de multiples commentateurs, à l'instar de Pickering, ont défini l'aporie centrale de la sociologie durkheimienne par la circularité qu'elle instaure entre catégories logiques et morphologie sociale. Dans les termes mêmes de Pickering, «la structure sociale explique les concepts; les concepts expliquent la structure sociale $e^{35} »$. Durkheim resterait donc prisonnier d'un raisonnement par renvoi infini entre le matérialisme de la référence au groupe social «naturel» et «réel», d'une part et, d'autre part, l'idéalisme des représentations collectives avec leur efficacité spécifique. En réponse à cette aporie, le symbolisme pourrait apparaitre comme le tiers manquant, la médiation qui seule peut rendre compte sans réductionnisme de la spécificité ontologique du social, et ainsi remédier au problème évoqué du dualisme. D'ailleurs, selon l'analyse classique de Parsons, Durkheim aurait contribué à définir une sorte de "réalisme symbolique", permettant d'indiquer une sortie possible du problème de la représentation, laquelle notion reste toujours comme engluée dans le dualisme sujet/objet. Le sens renouvelé qui est donné par Durkheim au symbolisme laisse effectivement entendre, pour reprendre les termes de Tarot, que «la société ne préexiste peut-être pas complètement à la symbolisation mais se crée et se fixe sur elle ${ }^{36} »$. De ce fait, la symbolisation n'est plus seulement la projection sur le monde d'un cadre social préexistant dans la morphologie réelle, mais «le processus par lequel un groupe en relation avec le monde se crée non seulement en fixant ses représentations, mais aussi les crée et les fixe en les échangeant sous des formes sensibles ${ }^{37}$ ».

Cette interprétation des thèses durkheimiennes a trouvé un écho, outre Tarot et Karsenti, chez d'autres exégètes, à l'instar de Paoletti, qui tente lui aussi de soutenir «la genèse sociale des catégories de la pensée sans abou-

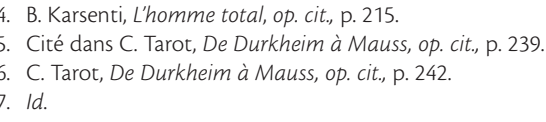


tir à une position relativiste ${ }^{38}$ ", et ainsi d'échapper à l'opposition classique entre rationalisme universaliste et constructivisme particulariste qui structure les positions dans le champ théorique des sciences sociales. Selon Paoletti, les arguments épistémologiques de Durkheim - sur la présentification matérielle de cet objet fuyant qu'est la société ou sur la nécessité de constamment revivifier le sentiment d'appartenance - débouchent in fine sur une thèse ontologique plus ou moins explicite. Celle-ci consiste justement à affirmer la réalité symbolique de la société, mêlant inextricablement matériel et idéel, et les englobant dans un esprit objectif donateur de sens et de signification. Car l'interaction entre individus, à partir de laquelle la réalité sociale se voit d'abord appréhendée, n'est possible que grâce à des intermédiaires matériels affectés de valeur, dont ces éléments tant sensibles qu'intelligibles que sont les symboles.

Toujours liés en quelque sorte à la sensibilité (d'où leur "matérialité»), qu'elle soit visuelle, auditive, tactile ou autre, les symboles ne se bornent donc pas à exprimer les représentations collectives, mais contribuent à les faire, à les transformer et à les transmettre. Il s'agit, en d'autres termes, d'une authentique fonction productive du symbolisme, et pas seulement communicationnelle, telle que le comprendra à son tour de façon réductionniste un Lévi-Strauss, y voyant surtout des modalités d'échange et de réciprocité. Dans le sillage de Durkheim, puis de Mauss, la fondation symbolique du social ne se réfère pas d'abord à un système de classification, du moins pas uniquement; car elle s'attache également à ériger une dimension partielle du réel en incarnation de ce qui ressort de plus sacré pour l'existence d'une société particulière ${ }^{39}$, délimitée en tant qu'identité dans le temps et l'espace, fût-ce de manière contingente et évolutive.

\section{Conclusion}

Considérant cette conception théorique de la constitution symbolique de la société, reste une question, en guise d'ouverture conclusive: la sociologie, en son fondement ultime, n'est-elle pas vouée à devoir rester fondamentalement durkheimienne? Au-delà des multiples discussions, oppositions ou interprétations de toutes sortes à propos d'une œuvre à la fois multiforme et

38. Giovanni Paoletti, «Durkheim et le problème de l'objectivité: une lecture des Formes élémentaires de la vie religieuse», Revue française de sociologie, vol. 43, n³, 2002, p. 438.

39. C'était déjà le problème soulevé voici plus de 35 ans par le philosophe Vincent Descombes, qui invitait à retrouver derrière le «symbolique» défini par le structuralisme lévi-straussien - synonyme d'un outil conventionnel et signifiant apte à permettre l'échange et la négociation - «la réalité énigmatique du sacré», laquelle entérine une Loi symbolique, en lien avec les aspects les plus fondamentaux pour l'existence d'une société: Vincent Descombes, «L'équivoque du symbolique», MLN, vol. 94, $n^{\circ}$ 4, French Issue: Perspectives in Mimesis, mai 1979 , p. 655-675. 
cohérente, débats qui témoignent de son infinie fécondité, la socio-anthropologie née au cœur de la modernité politique et économique possède une dimension indissolublement analytique et normative, ainsi que Durkheim l'a d'emblée énoncé. Par son orientation comparatiste et sa prudence conceptuelle, la sociologie durkheimienne permet de distinguer l'inscription sociale des catégories $-\mathrm{y}$ compris celles qui nous concernent le plus et qui sont d'un intérêt supérieur pour l'existence même d'une société démocratique: individu, justice, égalité ou liberté, etc. - de leurs nécessaires implications politiques, tout en conservant la visée d'une totalité morale qui puisse articuler ces réalités au sein d'un monde commun, d'un universum concret ainsi que le nomme si justement Michel Freitag ${ }^{40}$.

Il nous semble donc que l'affirmation, dans l'œuvre durkheimienne, d'une socio-anthropologie qui ne craigne pas de poser la question ontologique de l'institution symbolique du social, reste d'une actualité indépassable, dans une période contemporaine où ne s'imposent plus que les grammaires du flux, du réseau, de l'hybridité, toutes adossées au "plan d'immanence» deleuzien ${ }^{41}$, sans jamais reconnaître le détour constitutif de l'expérience sociale par une verticalité transcendante et objective qui seule est à même de la fonder dans la durée. De toute évidence, les intuitions durkheimiennes sur le symbolisme avancées dans les Formes élémentaires doivent être complétées et approfondies par les recherches de son neveu et disciple Marcel Mauss, ainsi que par les travaux d'auteurs contemporains comme Freitag, Dumont, Castoriadis, Gauchet, Sahlins, Descombes, Chanial, Flahaut, qui à divers titres, refusent de sacrifier le réalisme sociologique sur l'autel de la postmodernité triomphante. Le nom de Durkheim, néanmoins, pourrait bien servir de totem à ce clan, pour lequel le concept de «société» reste non seulement une référence historique, mais également une réalité à défendre.

40. Michel Freitag, L'abîme de la liberté, Montréal, Liber, 2011. Ce terme doit être rapproché de la notion d'« universel concret» évoquée par Louis Dumont dans ses travaux d'anthropologie comparative. Voir à ce sujet: Stéphane Vibert, Louis Dumont - Holisme et modernité, Paris, Michalon, coll. «Le bien commun», 2004. Pour une actualisation remarquable de cette perspective dumontienne, voir l'ouvrage collectif: André Iteanu (dir.), La cohérence des sociétés. Mélanges en hommage à Daniel de Coppet, Paris, Éditions de la MSH, 2010.

41. Voir l'ouvrage collectif de Jonathan Roberge, Yan Sénéchal et Stéphane Vibert (dir.), La fin de la société - Débats contemporains autour d'un concept classique, Montréal, Athéna éditions, 2012 